\title{
Performance Analysis of MIMO Systems over AWGN Channel with Antenna Selection using Zero Forcing Receivers
}

\author{
Navjot Kaur \\ Lovely Professional University \\ Phagwara, India
}

\author{
Lavish Kansal \\ Lovely Professional University \\ Phagwara, India
}

\begin{abstract}
High data rates within the limited radio frequency (RF) spectrum is always desirable that leads to radios with capabilities beyond a single-input single-output (SISO) topology. Recently introduced wireless systems have adopted multiple-input multiple-output (MIMO) topologies that use two or more transmitters and two or more receivers to send data simultaneously over the same RF bandwidth. The performance of MIMO system can be improved by using multiple antennas at transmitter and receiver so as to provide spatial diversity. In this paper, the performance analysis of MIMO system over AWGN fading channel with ZF receiver is presented. The effects of the antenna selection can also be analyzed from the simulated results. The BER (Bit Error Rate) performance characteristics of Zero-Forcing (ZF) receiver is investigated for M-PSK modulation technique over the AWGN channel.
\end{abstract}

Keywords -MIMO, AWGN, spatial diversity, BER, ZF, M-PSK, SNR, multi-path fading, STBC.

\section{INTRODUCTION}

Multiple antennas employed at transmitter and receiver sides are used in wireless communication so as to achieve the high data rates through spatial multiplexing [1]. MIMO involves multiple transmitters sending unique data content to multiple receivers using spatial multiplexing. MIMO does increase data rates and requires better SNR than an equivalent SISO transmission. This method offers higher capacity to wireless systems and the capacity increases linearly with the number of antennas.

Future trends of wireless communications mentioned below leads to MIMO development:-

- Future wireless applications create insatiability as demand for "high data rate" and "high link quality" wireless access has increased a lot.

- Spectrum has become a scarce and expensive resource as bandwidth is very limited

- Regulation, device and system capacity concerns as transmit power is limited

- Time and frequency domain processing are at limits, but space is not!

A MIMO system utilizes spatial diversity by using spatially separated antennas in a dense multipath scattering environment [2]. In MIMO, phased sets of antennas take advantage of the differences in the spatial propagation paths to improve signal robustness or to send multiple data sets over a single frequency band. In general, having multiple antennas offers three potential use cases: diversity, beam forming, and space division multiplexing (SDM). MIMO systems are implemented to obtain a diversity gain to combat signal fading.

An efficient implementation of space-time block coding (STBC) for broadband wireless communications improves the performance and diversity gains of a space time (ST) coding system through a number of parameters including type of trellis codes and channel fading[3][4]. The two main functions of STC: diversity \& multiplexing. The maximum performance needs tradeoffs between diversity and multiplexing. V-BLAST is a system in which full spatial diversity is usually not achieved [5].

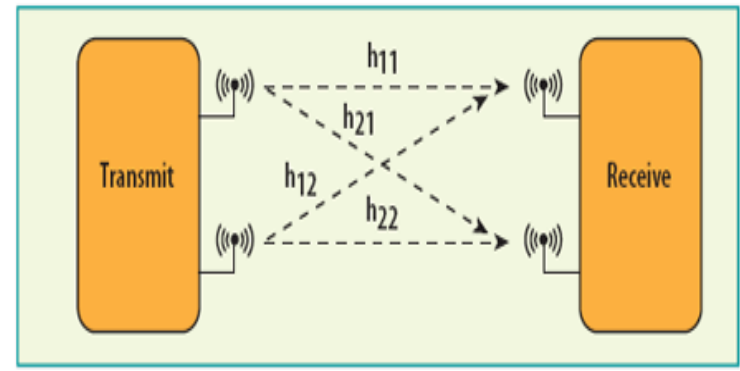

Fig. 1.1: Block Diagram of 2X2 MIMO System

The block diagram of MIMO system is shown in the Fig. 1.1. The basic main idea of MIMO is to improve BER or data rate (bits/sec) by using multiple TX/RX antennas. [6]. For achieving high data rate or improved BER, the core scheme used in MIMO system is space-time coding (STC).

MIMO provides higher capacity (bits/s/Hz), better transmission quality (BER), increased coverage and improved user position estimation due to the following factors:

- Spatial multiplexing gain : Capacity gain at no additional power or bandwidth consumption obtained through the use of multiple antennas at both sides of a wireless radio link

- Diversity gain : Improvement in link reliability obtained by transmitting the same data on independently fading branches

- Array gain

- Interference reduction

In this paper, the effects of AWGN channel are considered on the performance of MIMO systems with different antenna selection using ZF receivers. AWGN channel is a universal channel which adds a white Gaussian noise to the signal 
passing through it. It is a channel model used for analyzing the various modulation schemes.

\section{LITERATURE REVIEW}

Multiple input-multiple output (MIMO) communication systems employing coding techniques appropriate to multiple antenna transmissions have recently been embraced as an effective means to achieve high data rate over wireless channels.

A. I. Sulyman [7] describes the impact of antenna selection on the performance of multiple input-multiple output (MIMO) systems over nonlinear communication channels. The author has derived exact analytical expressions for evaluating the PWEP performance of space-time trellis codes over nonlinear MIMO channel.

C. Wang [8] explains the approach to increase the capacity of MIMO systems by employing the spatial multiplexing where independent information streams are transmitted from the antennas. These information streams are then separated at the receiver by means of appropriate signal processing techniques such as maximum likelihood (ML) which achieves optimal performance or linear receivers like Zero-Forcing (ZF) which provide sub-optimal performance but it also offers significant computational complexity reduction with tolerable performance degradation

The comparison of MIMO with SISO technology was discussed by S. G. Kim et. al [9]. MIMO can not only improve spectral efficiency, but also enhance link throughput or capacity of the system. The authors presented a tight closed form BER approximation of MPSK for MIMO ZF receiver over continuous flat fading channels. The larger the difference between the number of transmit antennas and the number of receive antennas is, the better performance is.

A simple two-branch transmit diversity scheme was presented by S. Alamouti [10]. The scheme uses two transmit antennas and one receive antenna. It provides the same diversity order as maximal-ratio receiver combining (MRRC) with one transmit antenna, and two receive antennas.

V. Tarokh et.al [11] design a channel codes for improving the high data rate and the reliability of communications over fading channels using multiple transmit antennas. Data is encoded by a channel code and the encoded data is split into multiple streams that are simultaneously transmitted using multiple transmit antennas. The received signal at each receive antenna is a linear superposition of the multiple transmitted signals perturbed by noise.

The performance analysis of the low-cost and effective transmission strategy that employs the simple spatial multiplexing at the transmitter and zero-forcing processing at the receiver in multiuser MIMO scheduling systems was discussed by C. Chen [12].

N. S. Kumar et. al [13], investigated about the three types of equalizer for MIMO wireless receivers. The authors discussed about a fixed antenna MIMO antenna configuration and compare the performance with all the three types of equalizer based receiver namely $\mathrm{ZF}, \mathrm{ML}$, and MMSE. BER performance of ML Equalizer is superior than zero forcing
Equalizer and Minimum Mean Square Equalizers. Based on the mathematical modeling and the simulation result it is inferred that the ML equalizer is the best of the three equalizers.

\section{MODULATION TECHNIQUE}

The digital communication system consists of two fundamentals components i.e. modulation and encoder. Modulation is the process of mapping the digital information to analog form so it can be transmitted over a long distance via channel. Modulator is a device used in transmitter side to perform modulation and demodulation is done at the receiver end by making use of demodulator. Demodulation is the inverse process of modulation so as to recover the transmitted digital information.

Phase-shift keying (M-PSK) for which the signal set is:

$\mathrm{X}_{\mathrm{i}}(\mathrm{t})=\frac{\sqrt{2 \mathrm{E}_{\mathrm{s}}}}{\mathrm{T}_{\mathrm{s}}} \cos \left(2 \pi * \mathrm{f}_{\mathrm{c \tau}}+\frac{2(\mathrm{i}-1)}{\mathrm{M}}\right) \quad \mathrm{i}=1,2, \ldots . \mathrm{M} \& 0<$ $t<\mathrm{T}_{\mathrm{s}}$

where $E_{s}$ the signal energy per symbol $T_{s}$ is the symbol duration and $\mathrm{f}_{\mathrm{c} \tau}$ is the carrier frequency.

This phase of the carrier takes on one of the $\mathrm{M}$ possible values.

$\theta=2(i-1)^{\pi / M} \quad i=1,2, \ldots . M$

M-ary PSK modulation yields a circular constellation as shown in Fig. 1.2. The main constraint was to keep the amplitude of the transmitted signals be constant.

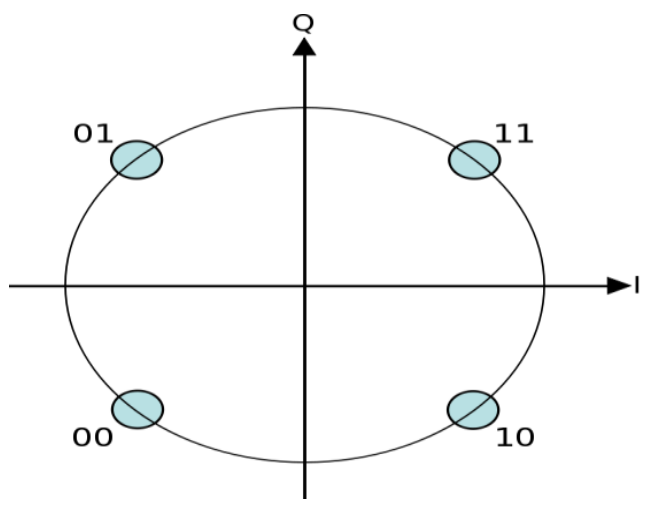

Fig. 1.2: Signal Constellation Diagram for 4-PSK

\section{AWGN CHANNEL}

AWGN channel is a channel that adds a white Gaussian noise to the signal passing through it. This implies that the channel's amplitude frequency response is flat (thus with unlimited or infinite bandwidth) and phase frequency response is linear for all frequencies so that modulated signals pass through it without any amplitude loss and phase distortion of frequency components. Fading does not exist. The only distortion is introduced by the AWGN.

$$
\begin{aligned}
& \text { The received signal is simplified to } \\
& \qquad \mathrm{r}(\mathrm{t})=\mathrm{x}(\mathrm{t})+\mathrm{n}(\mathrm{t})
\end{aligned}
$$

where $n(t)$ is the additive white Gaussian noise.

The whiteness of $\mathrm{n}(\mathrm{t})$ implies that it is a stationary random process with a flat power spectral density (PSD) for all frequencies. It is a convention to assume its PSD as 


$$
\mathrm{N}(\mathrm{f})=\mathrm{No} / 2,-\infty<f<\infty
$$

This implies that a white process has infinite power. This of course is a mathematical idealization. According to the Wiener-Khinchine theorem, the autocorrelation function of the AWGN is

$$
\mathrm{R}(\tau)=\mathrm{No} / 2 \delta(\tau)
$$

where $\delta(\tau)$ is the Dirac delta function.

\section{MIMO SYSTEM MODEL}

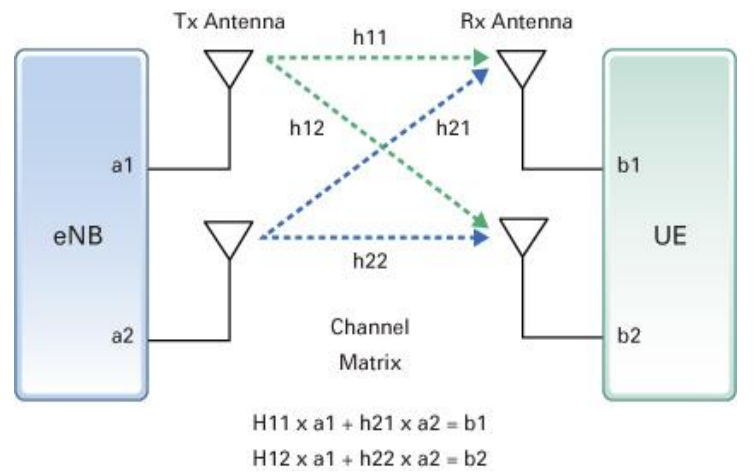

Fig. 1.3: The MIMO Channel

The 2X2 MIMO channel is represented in Fig. 1.3 with an antenna array with 2 elements at the transmitter and an antenna array with 2 elements at the receiver is considered. The input-output notation of the MIMO system can now be expressed by the equation:

$$
y(t)=H(\tau, t) \otimes s(t)+u(t) \quad(1.6)
$$

where $\otimes$ denotes convolution, $s(t)$ is a $n_{t} X 1$ vector corresponding to the $n_{t}$ transmitted signals, $y(t)$ is a $n_{r} X 1$ vector corresponding to the $\mathrm{n}_{\mathrm{r}}$ and $\mathrm{u}(\mathrm{t})$ is the additive white noise.

The impulse response of the channel between the $\mathrm{j}^{\text {th }}$ transmitter element and the $\mathrm{i}^{\text {th }}$ receiver element is denoted as $h_{i j}(\tau, t)$. The MIMO channel can then be described by the $n_{r} X$ $\mathrm{n}_{\mathrm{t}} \mathrm{H}(\tau, \mathrm{t})$ matrix:

$$
\mathrm{H}(\tau, \mathrm{t})=\left[\begin{array}{c}
\mathrm{h}_{1,1}(\tau, \mathrm{t}) \\
\mathrm{h}_{2,1}(\tau, \mathrm{t}) \\
\vdots \\
\mathrm{h}_{\mathrm{n}_{\mathrm{r}_{\mathrm{m}} \mathrm{n}_{1}}}(\tau, \mathrm{t})
\end{array}\right.
$$

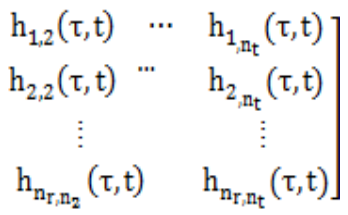

The matrix elements are complex numbers that correspond to the attenuation and phase shift that the wireless channel introduces to the signal reaching the receiver with delay $\tau$.

\section{ZERO FORCING EQUALIZER}

Zero Forcing Equalizer is a linear equalization algorithm which inverts the frequency response of the channel used in communication systems.

The Zero-Forcing Equalizer applies the inverse of the channel to the received signal, to restore the signal before the channel. The name Zero Forcing corresponds to bringing down the ISI to zero in a noise free case. This will be useful when ISI is significant compared to noise [8] [9].

For a channel with frequency response $F(f)$ the zero forcing equalizer $\mathrm{C}(\mathrm{f})$ is constructed such that $\mathrm{C}(\mathrm{f})=1 / \mathrm{F}(\mathrm{f})$. Thus the combination of channel and equalizer gives a flat frequency response and linear phase $\mathrm{F}(\mathrm{f}) \mathrm{C}(\mathrm{f})=1$. By using the linear model the received vector can be represented as:

$$
\mathrm{y}=\mathrm{Hx}+\mathrm{n}
$$

For a 2x2 MIMO channel, the channel is modeled as,

$$
\left(\begin{array}{l}
y_{1} \\
y_{2}
\end{array}\right)=\left(\begin{array}{ll}
h_{1,1} & h_{1,2} \\
h_{2,1} & h_{2,2}
\end{array}\right)\left(\begin{array}{l}
x_{1} \\
x_{2}
\end{array}\right)+\left(\begin{array}{l}
n_{1} \\
n_{2}
\end{array}\right)
$$

\section{SIMULATED RESULTS}

In this section, BER (Bit Error Rate) analysis of MIMO system over AWGN channel using STBC code structure is done for M-PSK Modulation techniques. The BER analysis of MIMO system is done for M-PSK over AWGN fading channel where $\mathrm{M}$ can be 32, 64, 128, 256, 512 and 1024 for different antenna configurations. Here receiving antennas used are ranging from $\mathrm{NR}=1$ to $\mathrm{NR}=4$.

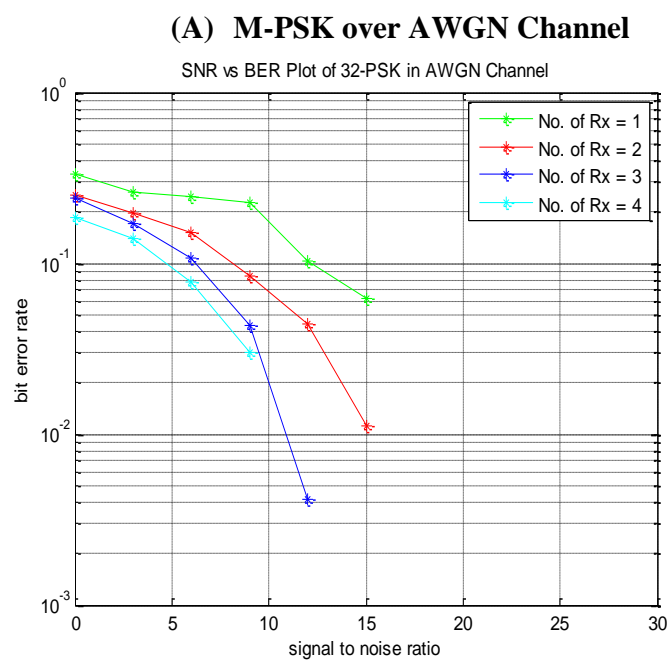

(a) 32-PSK 


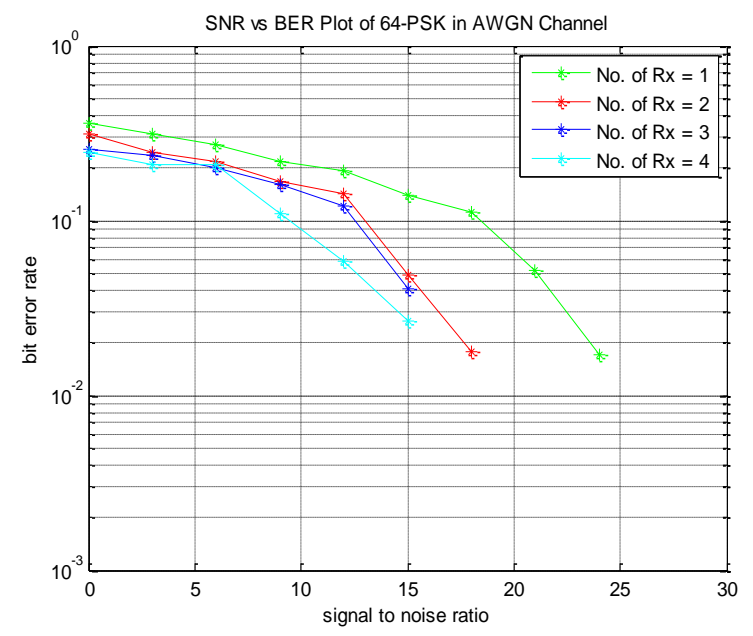

(b) 64-PSK

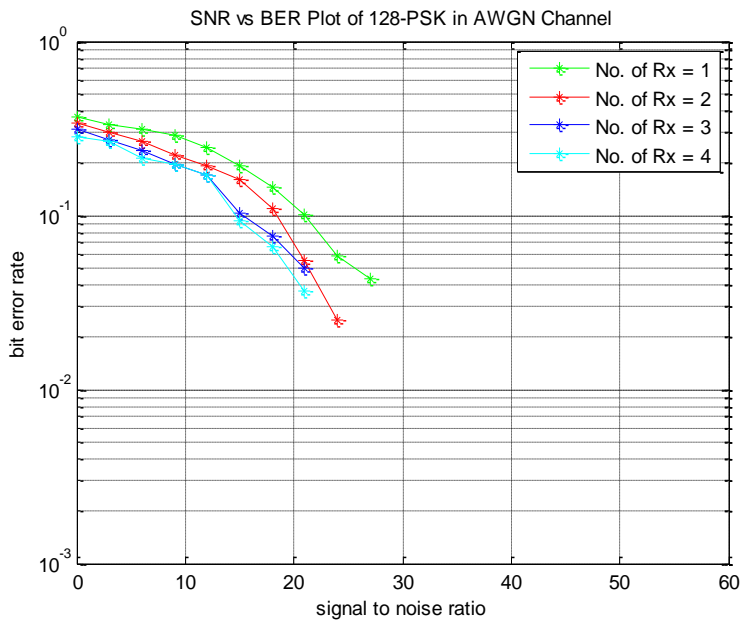

(c) 128-PSK

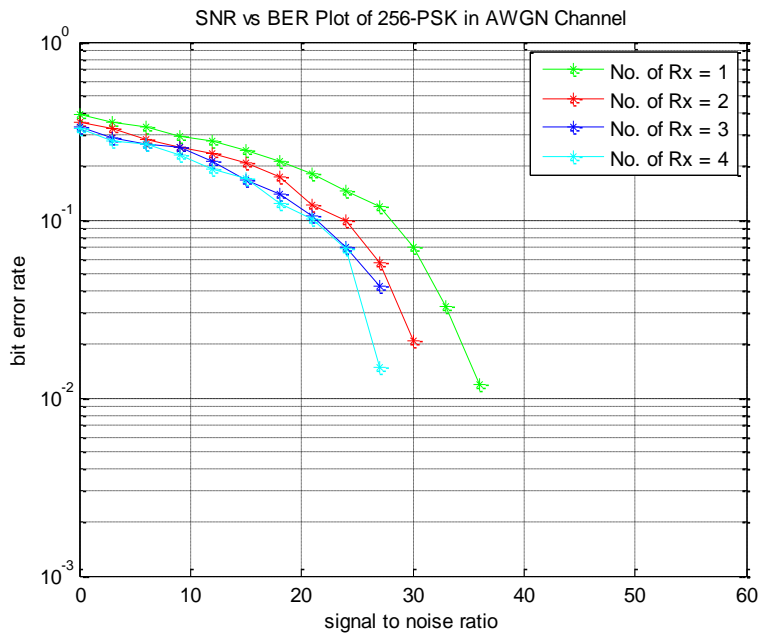

(d) 256-PSK

\section{CONCLUSION}

In this paper, the performance analysis of MIMO system over AWGN fading channel employing different antenna configurations is presented. It can be depicted from the graphs that the BER keeps on decreasing in MIMO system due to space diversity as increasing the number of receiving antennas. Here receiving antennas have been used ranging

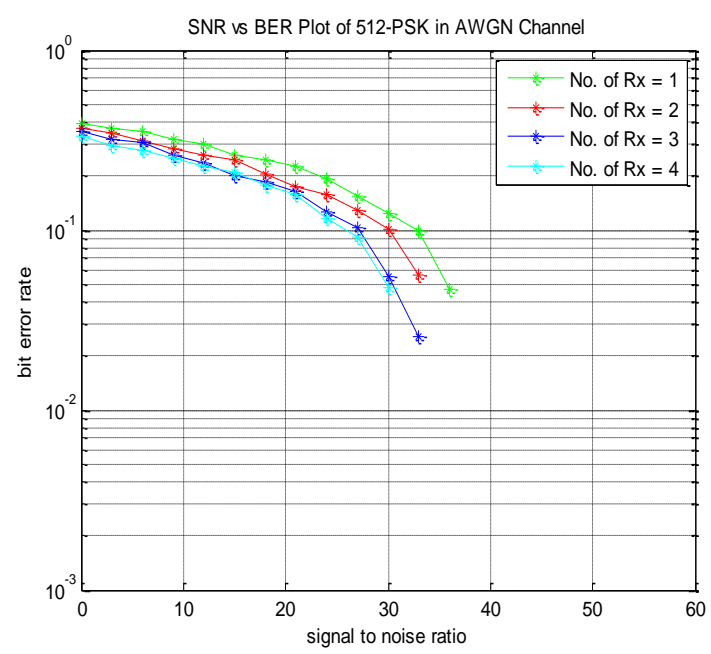

(e) 512-PSK

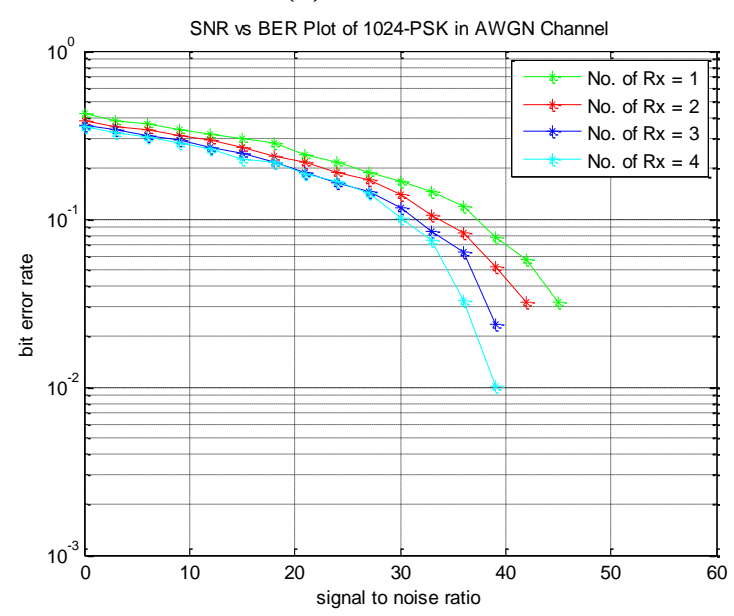

(f) 1024-PSK

Fig. 1.4: SNR vs BER plots for M-PSK over AWGN channel

In Fig. 1.4 (a) - (f), SNR vs. BER plots for M-PSK over AWGN channel for MIMO system with different antenna configurations have been presented. From the graphs, it can be concluded that as the number of receiving antennas are increasing in the MIMO system, the BER keeps on decreasing due to space diversity. Thus the system provides better BER performance over the AWGN fading channel.

from $\mathrm{NR}=1$ to $\mathrm{NR}=4$. Spatial diversity techniques are employed to improve signal quality and coverage. The multiple receivers or multiple transmitters reduce multipath fading and enhance SNR. Thus the proposed system provides better BER performance. 


\section{REFERENCES}

[1] R. W. Heath, "Multimode antenna selection for spatial multiplexing systems with linear receivers", IEEE Transactions on Signal Processing, Vol. 53, Issue 8, pp. 3042-3056, 2005.

[2] E. Casas \& C. Leung, "Performance of OFDM/FM scheme for data transmission over fading mobile radio channels", 36th IEEE Vehicular Technology Conference, Vol. 36, Issue 5, pp 103-108, 1986, Dallas, Texas.

[3] V. Tarokh, H. Jafarkhani \& A. R. Calderbank, "Spacetime block codes from orthogonal designs", IEEE Transactions on Information Theory, Vol. 45, Issue 5, pp. 1456-1467, 1999.

[4] G. Ganesan \& P. Stoica. 2001. "Space-time block codes: a maximum SNR approach", IEEE Transactions on Information Theory, Vol. 47, Issue 4, pp. 1650-1656, 2001 .

[5] P. W. Wolniansky, G. J. Foschini, G. D. Golden \& R. A. Valenzuela, "V-Blast: An architecture for realizing very high data rates over the rich-scattering channel", International Symposium on Signals, Systems and Electronics, pp. 295-300, 1998.

[6] X. Zhang, Z. Lv \& W. Wang, "Performance Analysis of Multiuser Diversity in MIMO Systems with Antenna Selection", IEEE Transactions on Wireless Communications, Vol. 7, Issue 1, pp. 15-21, 2008.

[7] A. I. Sulyman, "Performance of MIMO Systems With Antenna Selection Over Nonlinear Fading Channels", IEEE Journal of Selected Topics in Signal Processing, Vol. 2, Issue 2, pp. 159-170, 2008.
[8] C. Wang, "On the Performance of the MIMO ZeroForcing Receiver in the Presence of Channel Estimation Error", IEEE Transactions on Wireless Communications, Vol. 6, Issue 3, pp. 805 - 810, 2007.

[9] S. G. Kim, D. Yoon, Z. Xu \& S. K. Park, "Performance Analysis of the MIMO Zero-Forcing Receiver over Continuous Flat Fading Channels", IEEE Journal of Selected Areas in Communications, Vol. 20, Issue 7, pp. $324-327,2009$

[10] S. Alamouti, "A simple transmit diversity technique for wireless communications", IEEE Journal on Selected Areas of Communication, Vol. 16, Issue 8, pp. 1451$1458,1998$.

[11] V. Tarokh, N. Seshadri \& A. R. Calderbank, "SpaceTime Codes for High Data Rate Wireless Communication: Performance Criterion and Code Construction", IEEE Transactions on Information Theory, Vol. 44, Issue 2, pp. 744-765, 1998.

[12] C. Chen, "Performance Analysis of Scheduling in Multiuser MIMO Systems with Zero-Forcing Receivers", IEEE Journal of Selected Areas in Communications, Vol. 25, Issue 7, pp. 1435-1445, 2007

[13] N. S. Kumar, G. J. Foschini, G. D. Golden \& R. A. Valenzuela, "Bit Error Rate Performance Analysis of ZF, ML and MMSE Equalizers for MIMO Wireless Communication Receiver", European Journal of Scientific Research, Vol. 59, Issue 4, pp. 522-532, 2011. 